\title{
A $\beta$ Ti-20Nb-10Ta-5Zr Alloy with the Surface Structured on the Micro- and Nanoscale
}

\author{
M. A. Sevostyanov ${ }^{a}$, A. S. Baikin ${ }^{a}$, M. A. Kaplan ${ }^{a}$, A. G. Kolmakov ${ }^{a}$, S. V. Gudkov ${ }^{b}$ M. B. Rebezov ${ }^{b, c, *}$, \\ and Corresponding Member of the RAS S. V. Garnov ${ }^{b, * *}$
}

Received November 30, 2020; revised November 30, 2020; accepted December 28, 2020

\begin{abstract}
This alloy is shown to be homogeneous ( $\mathrm{Ti} 65 \%, \mathrm{Nb} 20 \%$, Ta 10\%, $\mathrm{Zr} 5 \%$ ). A change in the elemental composition is observed only in the layer close to the surface with a thickness of about $100 \mathrm{~nm}$. The alloy surface is depleted in titanium $(\sim 20 \%)$ and enriched in tantalum $(\sim 20 \%)$. There is also a large amount of oxides on the surface $(\sim 50 \%)$. The alloy is single-phase with a $\beta$-Ti-type crystal lattice (cubic system, space group $\operatorname{Im} 3 \mathrm{~m}$ ). The alloy has yield strength of about $550 \mathrm{MPa}$ and a tensile strength of about $700 \mathrm{MPa}$. The Young's modulus is about $50 \mathrm{GPa}$. The relative elongation of the alloy is about $1.4 \%$. On a microscale, folds and longitudinal comb-like structures up to $0.5 \mu \mathrm{m}$ in height are found on the surface of wires and plates made of the $\mathrm{Ti}-20 \mathrm{Nb}-10 \mathrm{Ta}-5 \mathrm{Zr}$ alloy. When analyzing nanotopology, it is found that, even between comblike structures or at their tops, there are irregularities up to $100-150 \mathrm{~nm}$ in height.
\end{abstract}

Keywords: titanium alloy, nanotopology, optical micrograph, microstructural analysis

DOI: $10.1134 /$ S1028335821010079

The search for materials to use for medical implants has already been underway for more than two centuries [1]. Unfortunately, known materials, especially metals, are not indifferent to the human body [2]. Historically, titanium was one of the first metals that was used for medical implants [3]. It was used due to its low density, high corrosion resistance, fast passivation, and ability to form stable oxides. Nowadays, titanium is often replaced by its alloys. One of the first such alloys was nitinol. Nitinol, an alloy of nickel and titanium, was first obtained in the 1960s during the development of a thermal protection coating in a naval laboratory [4]. Nitinol later began to be used effectively in surgery, cardiology, dentistry, radiology, and devices for various purposes [5]. Such a variety of medical applications is associated with the unique properties of nitinol, more precisely, the shape-memory effect and superelasticity. The cost for these properties was the high toxicity of nickel. Currently, work is under way to create titanium alloys with the shape-

\footnotetext{
${ }^{a}$ Baikov Institute of Metallurgy and Materials Science, Russian Academy of Sciences, Moscow, 123456 Russia

${ }^{b}$ Prokhorov General Physics Institute of the Russian Academy of Sciences, Moscow, 119991 Russia

${ }^{c}$ Razumovskii Moscow State University of Technologies and Management (First Cossack University), Moscow, 109004 Russia

*e-mail:rebezov@yandex.ru

**e-mail:svgarnov@mail.ru
}

memory effect and superelasticity. In such alloys, one tries to replace nickel with less toxic metals [6-10]. A large number of titanium alloys are known to have the desired characteristics. The surface structure affects a number of alloy properties, especially those related to the mechanical properties and biocompatibility. In this study, we obtained and investigated the $(\beta) \mathrm{Ti}-$ $20 \mathrm{Nb}-10 \mathrm{Ta}-5 \mathrm{Zr}$ alloy with structured surfaces.

The alloy of $\mathrm{Ti}-20 \mathrm{Nb}-10 \mathrm{Ta}-5 \mathrm{Zr}$ (at \%) composition was obtained from iodide titanium, iodide zirconium, technically pure niobium, and technically pure tantalum. The sample was melted in an electric-arc vacuum furnace with a non-expendable tungsten electrode as described previously in [11]. The plates and wires with structured surfaces were obtained with a DUO-300 modified reversing mill and special polishing [12]. The X-ray diffractograms were obtained with an ARL X'TRA device ( $\mathrm{Cu} K_{\alpha}$ radiation) in the parallel-beam geometry. The electron-microscope investigations of the samples were carried out with a JEOL LSM-IT500 scanning electron microscope. The phase composition was investigated with a JEOL JAMP-9500F Auger-electron spectrometer. For calorimetric investigations, we used a differential scanning calorimeter Netzsch STA-409PC. For dilatometric investigations, a Netzsch DIL 402 C7G high-temperature dilatometer was used. The samples were visualized with an Olympus GX51 optical microscope. For investigating the surface topology at the micro- and nanolevel, we used a Nanopics 2000 atomic-force microscope and an MIM 321 laser interference-mod- 


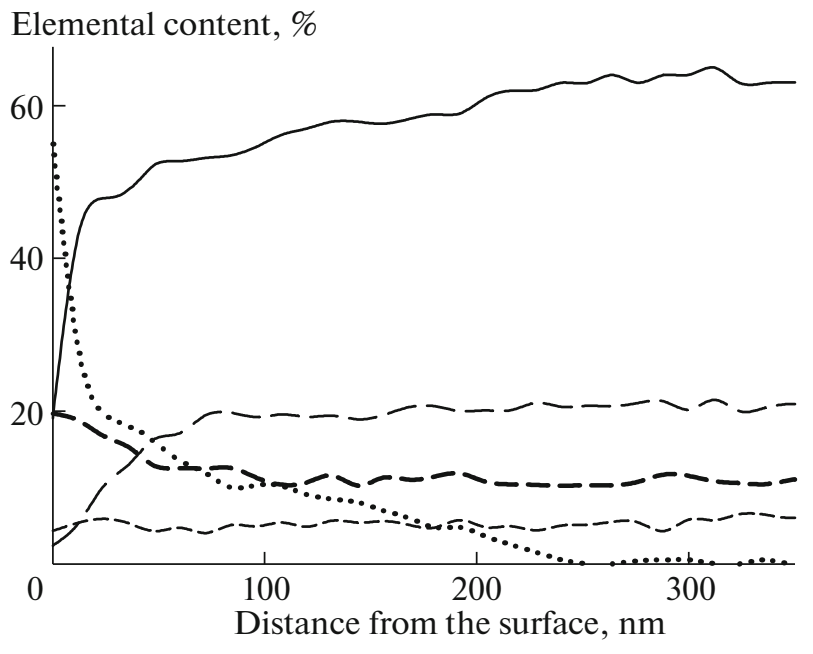

Fig. 1. Layer-by-layer elemental analysis of the $\mathrm{Ti}-20 \mathrm{Nb}-$ $10 \mathrm{Ta}-5 \mathrm{Zr}$ alloy.

ulation microscope. The mechanical properties were investigated with an Instron 3382 universal testing machine with a tensile speed of $1 \mathrm{~mm} / \mathrm{min}$.

We investigated the elemental composition of the alloy. It is shown that the elemental composition inside the alloy is relatively constant: $\mathrm{Ti} 65 \%, \mathrm{Nb} 20 \%$, Ta $10 \%$, and Zr 5\% (Fig. 1). The zirconium content on the alloy surface and inside the ingot is also relatively constant, whereas the alloy surface is depleted in titanium $(\sim 20 \%)$ and enriched in tantalum $(\sim 20 \%)$. A large amount of oxides $(\sim 50 \%)$ is present on the surface. The content of oxygen atoms decreases to $20 \%$ at a distance of $20 \mathrm{~nm}$ from the surface and to $10 \%$ at a distance of $100 \mathrm{~nm}$. At a distance of 250-300 $\mathrm{nm}$ from the surface, there are only trace amounts of oxygen atoms.

The phase composition of the TiNbTaZr alloy was investigated. All samples are found single-phase with a $\beta$-Ti-type lattice (cubic syngony, spatial group $\operatorname{Im} 3 \mathrm{~m}$ ). The lattice parameter is $3.30982 \pm 0.00007 \AA$. The peak positions are as follows: $38.6508,55.6833$, 69.6987, 82.5072, 94.9302, 107.5824, and 121.1965. The peak with the position of 107.5824 has a very low intensity of $0.41 \%$. It is shown that all samples are polycrystalline.

The mechanical tests of the alloy are carried out. It is shown that the yield strength of the alloy is $550 \mathrm{MPa}$, and the tensile strength is about $700 \mathrm{MPa}$. Young's modulus is about $50 \mathrm{GPa}$. The relative elongation of the alloy is about $1.4 \%$.

Calorimetric analysis of the alloy was carried out. The samples were investigated at temperatures from 400 to $900^{\circ} \mathrm{C}$ in an argon medium. The heating was carried out at the rate of $10^{\circ} \mathrm{C}$ per minute. The weight of the samples was $50 \mathrm{mg}$ each. It was shown that the phase transition was observed at a temperature just below $600^{\circ} \mathrm{C}$.
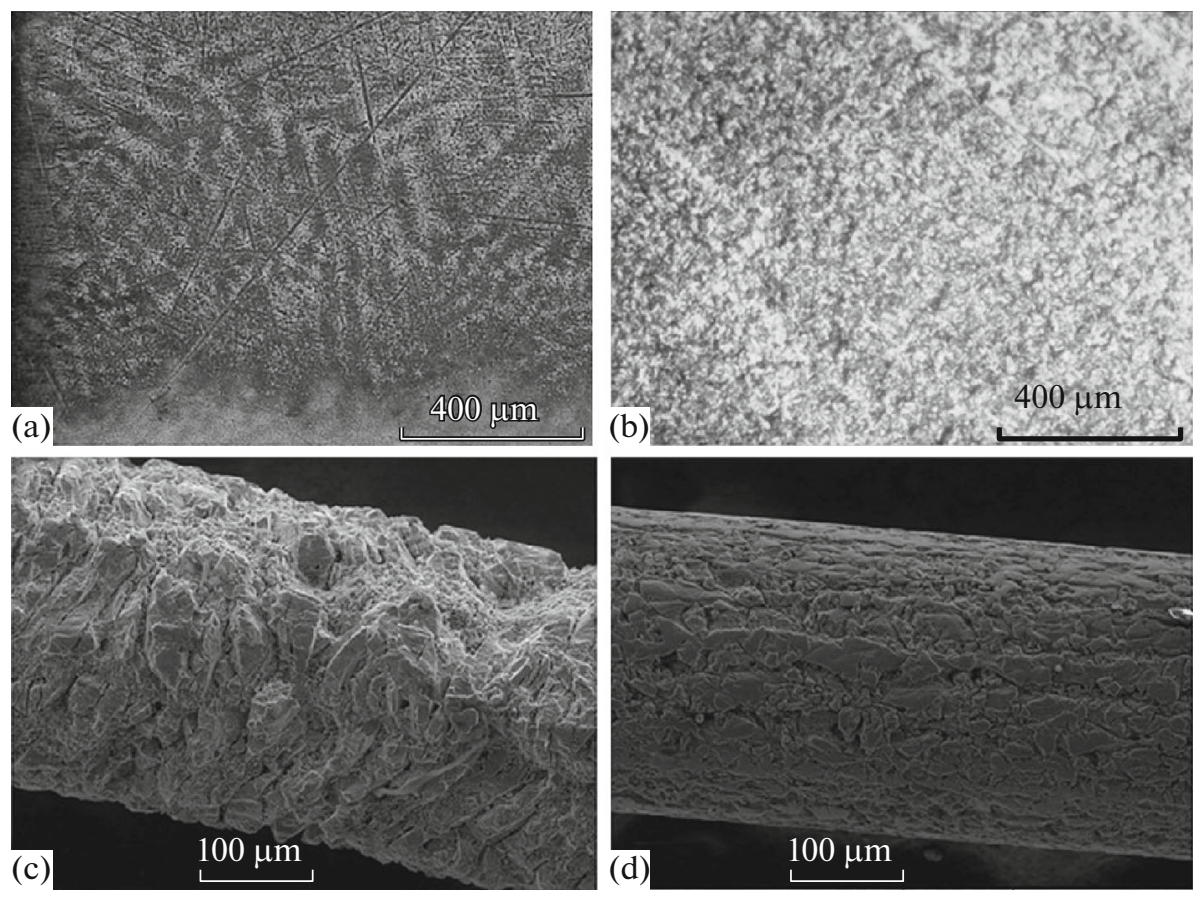

Fig. 2. Photographs of the Ti-20Nb-10Ta-5Zr-alloy surface. (a) Optical micrograph of the alloy surface after production; (b) optical micrograph of the alloy surface after plate fabrication and polishing; (c) photo of wire surface from the Ti-20Nb$10 \mathrm{Ta}-5 \mathrm{Zr}$ alloy after production (scanning electron microscopy); (d) photo of the wire surface from the $\mathrm{Ti}-20 \mathrm{Nb}-10 \mathrm{Ta}-5 \mathrm{Zr}$ alloy after polishing (scanning electron microscopy). 


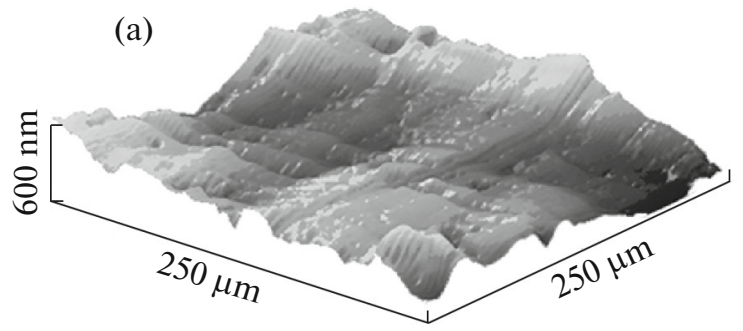

(b)

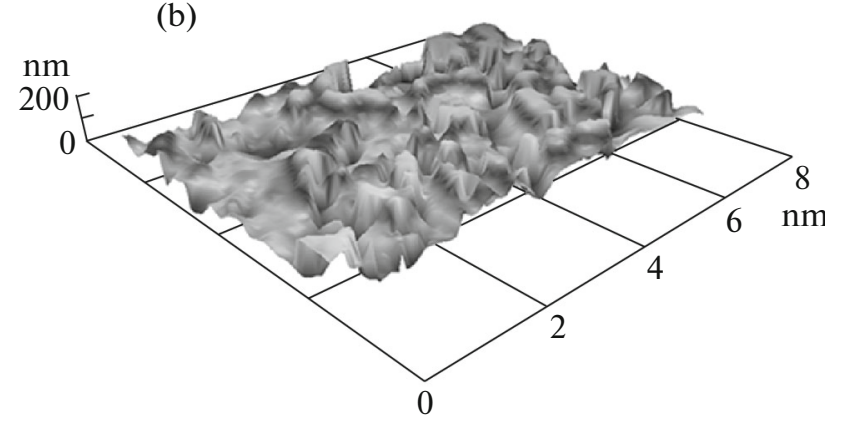

Fig. 3. Surface topology of the $\mathrm{Ti}-20 \mathrm{Nb}-10 \mathrm{Ta}-5 \mathrm{Zr}$ alloy: (a) investigation of the surface topology with the atomic force microscope; (b) investigation of the surface topology with the laser modulation-interference microscope.

The surface morphology of ingots made of the Ti$20 \mathrm{Nb}-10 \mathrm{Ta}-5 \mathrm{Zr}$ alloy was investigated. It was found that there are micrometer roughnesses on the surface of ingots (Fig. 2a). The wire obtained from the Ti$20 \mathrm{Nb}-10 \mathrm{Ta}-5 \mathrm{Zr}$ alloy also has a complex surface morphology (Fig. 2c). After structuring polishing, the microstructure of both the ingot (Fig. 2b) and the wire (Fig. 2d) contains less roughness. Part of the surface has no obvious defects. Such relatively smooth areas of the surface were examined by atomic-force microscopy and laser modulation-interference microscopy. It was shown that, even on relatively smooth areas, there are folds and longitudinal comb-like structures (Fig. 3a) up to $0.5 \mu \mathrm{m}$ in height. When analyzing the nanotopology, it was found that, even between the comb-like structures or on their tops, there are irregularities up to $100-150 \mathrm{~nm}$ high (Fig. 3b).

These data suggest that the alloy we obtained is homogeneous. The X-ray investigation confirms that it is the obtained crystal structure that is responsible for the appearance of superelasticity and the shapememory effect. At the same time, the tensile strength of the $\mathrm{Ti}-20 \mathrm{Nb}-10 \mathrm{Ta}-5 \mathrm{Zr}$ alloy turned out to be about $700 \mathrm{MPa}$, which is slightly higher than that of competitors. For example, it is $50 \mathrm{MPa}$ higher than that of $\mathrm{Ti}-33 \mathrm{Nb}-4 \mathrm{Sn}[13]$, and $100 \mathrm{MPa}$ higher than that of the Ti-25Ta alloy [14]. The Ti-20Nb-10Ta$5 \mathrm{Zr}$ alloy can be perfectly polished. After polishing, the surface of the alloy looks absolutely smooth in the microstructural analysis; there are no areas of crack formation. When passing to the nanoscale level, a pronounced relief is observed on the surface, but no cracks or zones of their formation are observed. On the whole, the mechanical and physicochemical properties suggest that the $\mathrm{Ti}-20 \mathrm{Nb}-10 \mathrm{Ta}-5 \mathrm{Zr}$ alloy can be used in biomedical and veterinary applications.

\section{FUNDING}

This work was supported by a grant for large scientific projects in the priority directions of scientific-technological development (agreement no. 075-15-2020-774).

\section{OPEN ACCESS}

This article is distributed under the terms of the Creative Commons Attribution 4.0 International Public License (http://creativecommons.org/licenses/by/4.0/), which permits unrestricted use, distribution, and reproduction in any medium provided you give appropriate credit to the original author(s) and the source, provide a link to the Creative Commons license, and indicate if changes were made.

\section{REFERENCES}

1. R. Langer and D. A. Tirrell, Nature (London, U.K.) 428, 487 (2004).

2. F. Matassi, A. Botti, L. Sirleo, C. Carulli, and M. Innocenti, Clin. Cases Miner. Bone. Metab. 10, 111 (2013).

3. A. Dehghanghadikolaei and B. Fotovvati, Materials 12, 1795 (2019).

4. W. Buehler and R. Wiley, Trans. Am. Soc. Met. 55, 269 (1962).

5. V. Ya. Erofeev and M. V. Kabanov, Dokl. Phys. 64, 53 (2019).

6. P. A. B. Kuroda, F. F. Quadros, K. S. J. Sousa, T. A. G. Donato, R. O. Araujo, and C. R. Grandini, J. Mater. Sci. Mater. Med. 31, 19 (2020).

7. M. Kaur and K. Singh, Mater. Sci. Eng. 102, 844 (2019).

8. S. V. Konushkin, K. V. Sergiyenko, E. O. Nasakina, V. G. Leontyev, O. G. Kuznetsova, D. D. Titov, et al., Mater. Chem. Phys. 255, 123557 (2020).

9. M. A. Sevostyanov, A. G. Kolmakov, K. V. Sergiyenko, M. A. Kaplan, and A. S. Baikin, J. Mater. Sci. 55, 14516 (2020).

10. E. O. Nasakina, S. V. Konushkin, M. A. Sudarchikova, K. V. Sergienko, A. S. Baikin, A. M. Tsareva, et al., Materials 13, 2187 (2020).

11. S. V. Gudkov, A. V. Simakin, M. A. Sevostyanov, S. V. Konushkin, M. Losertova, A. Yu. Ivannikov, et al., Metals 10, 1584 (2020).

12. S. V. Gudkov, A. V. Simakin, S. V. Konushkin, A. Yu. Ivannikov, E. O. Nasakina, L. A. Shatova, et al., J. Mater. Res. Tech. 9, 16018 (2020).

13. X. Cheng, S. Liu, C. Chen, W. Chen, M. Liu, R. Li, et al., J. Mater. Sci. Mater. Med. 30, 91 (2019).

14. Y. L. Zhou and M. Niinomi, Mater. Sci. Eng. C 29, 1061 (2009).

Translated by $V$. Bukhanov 